\title{
FODMAP-specific mobile application: impact on gut symptoms in 11,689 people, and dietary triggers in 2,053 people
}

\author{
Eirini Dimidi ${ }^{1}$, Kevin Whelan ${ }^{1}$ and Miranda CE Lomer ${ }^{1,2}$ \\ ${ }^{1}$ Department of Nutritional Sciences, King's College London, London, United Kingdom and \\ ${ }^{2}$ Guy's and St Thomas' NHS Foundation Trust, Department of Nutrition and Dietetics, London, United Kingdom
}

A diet low in fermentable carbohydrates (FODMAPs) is effective in improving functional bowel symptoms. Mobile/online access to accurate information about the low FODMAP diet is limited. Recent research showed that dietitians recommend apps to their patients to assist them while following a low FODMAP diet ${ }^{(1)}$. Although the need for personalised apps for the management and monitoring of chronic gastrointestinal disorders has been previously highlighted ${ }^{(2)}$, there is no research on users' experience of using such apps. The aim of this evaluation was to (i) assess self-reported gut symptoms during FODMAP restriction; and (ii) identify common dietary FODMAP triggers in users of the FODMAP by FoodMaestro app.

Data was collected from all app users who provided informed consent during FODMAP restriction and FODMAP reintroduction. The app provides education, guidance, lists of suitable/unsuitable foods for the low FODMAP diet during FODMAP restriction, and lists food challenges to identify dietary triggers during FODMAP reintroduction. Gut symptoms were recorded using a 4-point Likert scale (none, mild, moderate, severe) at baseline and at the end of FODMAP restriction and each day of a food challenge during FODMAP reintroduction. Dietary FODMAP triggers were identified from symptom response data to FODMAP food challenges. Symptom control during each challenge was assessed as a dichotomous outcome. Chi-square tests were used to analyse categorical data.

At baseline, data were available for 11,689 people who had moderate/severe overall abdominal symptoms, of whom $6,116(52 \%)$ still reported moderate/severe overall abdominal symptoms at the end of the FODMAP restriction stage, therefore the app was effective in reducing overall gut symptoms in $48 \%$. Significantly fewer users reported moderate/severe pain, bloating, flatulence, diarrhoea, and constipation at the end of FODMAP restriction compared to baseline (Figure 1).

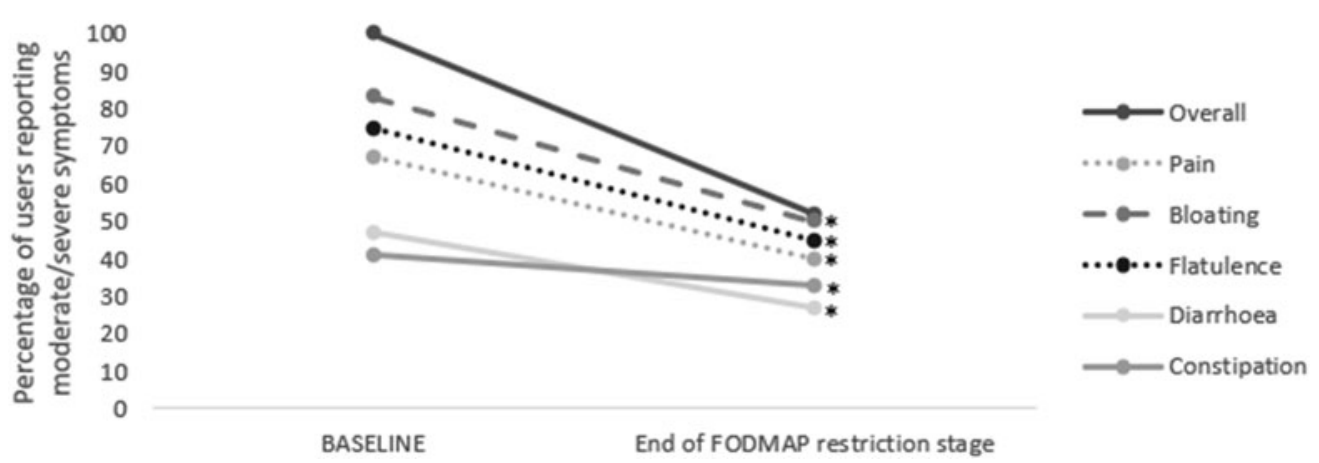

Fig. 1. Proportion of users reporting moderate/severe symptoms at baseline and end of FODMAP restriction $(n=11,689)$. ${ }^{*} \mathrm{p}<0.001$

During FODMAP reintroduction, 2,053 users completed at least one FODMAP food challenge, with 8,760 food challenges undertaken in total. The most frequently undertaken food challenge was wheat bread $(1,146 / 2,053,56 \%)$, followed by onion $(918 / 2,053$, $45 \%)$, garlic $(699 / 2,053,34 \%)$, milk $(687 / 2,053,33 \%)$, and wheat pasta $(548 / 2,053,27 \%)$. Of these who undertook these challenges, $474 / 1,146(41 \%)$ reported wheat bread as a dietary trigger of their symptoms, 359/918 (39\%) reported onion, $245 / 699(35 \%)$ reported garlic, $247 / 687(36 \%)$ reported milk, and 222/548 (41\%) reported wheat pasta.

This is the first study investigating the effectiveness of a mobile app to support people following the low FODMAP diet and shows that over half of users with symptoms before starting the diet report symptom improvement following FODMAP restriction. It identified the FODMAP food challenges that users choose to undertake during FODMAP reintroduction, as well as the self-reported tolerance levels. Randomised controlled trials are needed to establish whether mobile applications can improve gut symptoms and identify FODMAP dietary triggers in people with functional bowel disorders.

1. Chen et al. (2017) J Hum Nutr Diet 30(4), 439-52.

2. Riaz et al. (2016) Clin Gastroenterol Hepatol 14, 1697-705. 